\title{
Perceptions of Supervisors of Peer Support Workers (PSW) in Behavioral Health: Results from a National Survey
}

\author{
Dana Foglesong ${ }^{1,2} \cdot$ Amy B. Spagnolo $^{3}$ (1) $\cdot$ Rita Cronise $^{3} \cdot$ Joanne Forbes $^{3} \cdot$ Peggy Swarbrick $^{4} \cdot$ Jonathan P. Edwards $^{5}$. \\ Carlos Pratt ${ }^{3}$
}

Received: 28 December 2020 / Accepted: 12 May 2021 / Published online: 4 June 2021

(C) The Author(s), under exclusive licence to Springer Science+Business Media, LLC, part of Springer Nature 2021

\begin{abstract}
This content analysis of open-ended survey responses compares and contrasts perceptions on supervision from supervisors with experience providing direct peer support services (PS) and supervisors without experience providing direct peer support services (NPS).A 16-item online survey was distributed via the National Association of Peer Supporters (N.A.P.S.) listserv and through peer networks and peer run organizations. Responses from 837 respondents, across 46 US states, were analyzed. Four open ended questions assessed supervisors' perceptions on differences supervising peer support workers (PSW) as compared to other staff, important qualities of PSW supervisors, roles when supervising a PSW, and concerns about PSWs in the organization. Among NPS and PS, three major differences in themes emerged: the knowledge required of supervisors, understanding of the role of the PSW, and supervisors' beliefs regarding PSW competencies. PS have a more nuanced understanding of the peer support worker role and the impact of lived experience in the role.
\end{abstract}

Keywords Peer support worker - Supervision of peer support worker - Attitudes of supervisors of peer support workers · Role of the supervisor of peer support workers · Peer supervision

\section{Introduction}

Current literature on barriers to the inclusion of peer support workers (PSW) within mental health systems suggests that supervision is a key component to addressing challenges experienced while implementing peer support services (Delman \& Klodnick, 2016; Gates \& Akabas, 2007; Gates et al., 2010). Despite references to supervision as a possible solution to employment challenges, "supervision and the retention of peer providers do not appear in the literature

Amy B. Spagnolo

amy.spagnolo@shp.rutgers.edu

Magellan Complete Care, 691029, Orlando, FL 32869, USA

2 Southpark Center Loop, Bldg. 1200, Suite 250, Orlando, FL 32819, USA

3 Rutgers The State University of New Jersey, 675 Hoes Lane, Piscataway, NJ, USA

4 Rutgers University Behavioral Health, Collaborative Support Programs of NJ, 8 Spring Street, Freehold, NJ, USA

5 National Association of Peer Supporters (N.A.P.S.), Washington D.C, Was, USA as much as recruiting and hiring" (Jorgenson \& Schmook, 2014, p. 8). In 2007, the Centers for Medicare and Medicaid Services (CMS) published a guidance letter on the development of peer support services and their reimbursement under Medicaid requiring that supervision of PSW "must be provided by a competent mental health professional (as defined by the State)" (Smith, 2007). A decade later, a systematic review of the peer supervision literature concluded, "very little has been written on the topic of supervision" (Martin, Jordan, Razavi, \& Van Burnham, 2017, p.1). With the introduction of a non-clinical peer support workforce under the direct supervision of licensed mental health professionals without experience working as a PSW (NPS), a frequently cited issue in the literature is a lack of role clarity (Gates \& Akabas, 2007; Wolf et. al, 2010; Kuhn et. al, 2015; Jenkins et al, 2017). However, there is no evidence that role clarity issues exist within the delivery of peer support services in peer-run organizations that, by nature, have operated under an Apprenticeship Model of supervision with supervisors who themselves have been peer support providers (PS). This model has existed for several decades. This observation suggests that supervisors with the lived experience of having a disclosed mental health condition and work experience 
of providing peer support may have different perceptions about supervision of the PSW than those who do not have that shared experience.

Supervision as an apprenticeship for a field of practice is a time-honored tradition as old as the practice of medicine (Stephan \& Cheung, 2017). The Apprenticeship Model is described as an approach to supervision that occurs through a combination of observing, coaching, and practice. The Cognitive Apprenticeship Model shares the traditional apprenticeship's focus on learning complex tasks from experts, but further emphasizes cognitive skills of the teachers and learners, not as readily observable (Collins, 2006; Stalmeijer et al., 2013). The Apprenticeship Model of supervision has been applied in a variety of health disciplines such as certified behavioral analysis, music therapy, psychotherapy, and teacher education (Hartley et al., 2016; Feinstein et al., 2015; Donaldson, 2015). However, across the country, in the behavioral healthcare field, peer support workers (PSW) are being supervised by licensed professionals who have neither experienced nor provided peer support themselves, non-peer supervisors (NPS). This has resulted in a model of supervision that does not align with supervision as it is practiced in other areas of behavioral health care and defies the principles of an Apprenticeship Model. Furthermore, supervision can be viewed as a developmental process in which a less experienced practitioner (supervisee) gains experience, confidence, and competence under the direction of a more experienced practitioner (Stoltenberg, 2005). While NPS bring a multitude of experiences to the supervisory relationship, they lack the experience that peer support workers are expected and required to have.

The roles and responsibilities of the peer supervisor, approaches to supervision, and best practices for supervision have been discussed at conferences such as the 2014 Pillars of Peer Support summit convened specifically to address peer supervision (Daniels, Tunner, Powell, Fricks, $\&$ Ashenden, 2015, p. 10-12). Toolkits and guides to provide strategies for supervisors and managers to introduce peer support services into mental health agencies exist (Hendry, Hill, \& Rosenthal, 2014; Jorgenson \& Schmook, 2014; Mead, 2014; Philadelphia DBHIDS, 2017; Swarbrick, 2010; Smith, 2007; Tucker et al., 2013). Some provide practical tips and approaches for supervising peer support staff including the scope of practice, responsibilities, purpose of supervision, agency culture, and quality improvement strategies. Mead (2014), in particular, uses a mutual rather than hierarchical approach to supervision called co-reflection. While resources exist on supervision practices, analyses of NPS perceptions on supervision practices have not been reported in the literature.

The purpose of this analysis of open-ended survey responses collected from supervisors with experience working as a PSW (PS) and supervisors without experience working as a PSW (NPS) was to compare and contrast their perceptions on supervision. The findings also led the authors to propose a supervision model that accounts for lack of experiential knowledge. The survey found the current supervision of PSWs in behavioral healthcare is predominantly performed by licensed professionals who do not have experience in providing direct peer support services. Given the growth of peer support services in behavioral health, the trend for NPS supervision will continue to be the norm. Therefore, strategies that address the short comings that exist when a full apprenticeship model of supervision is not implemented are suggested.

\section{Methods}

\section{Sample}

Survey data was collected and analyzed from October 2017 to March 2018 via a 16-item online survey, Supervising Peer Support Workers in Behavioral Health Settings, developed by the Director of Recovery and Resiliency Services at Magellan Complete Care and distributed via the National Association of Peer Supporters (N.A.P.S.) listserv. Responses from 1238 respondents, across 46 US states, were collected.

Of the 1238 responses, respondents who were not currently supervising PSW were removed along with responses that were incomplete. The remaining 837 responses were analyzed including 638 supervisors who did not have experience working as a PSW and 199 supervisors who identified as having experience working as a PSW providing direct services. Respondents were able to report more than one license or credential and reported a variety of professional licenses and credentials including social work (MSW, LCSW, LICSW; $n=300$ ), licensed mental health counselor (LMHC, LPC, LMFC; $\mathrm{n}=134$ ), nurse (LPN, RN, BSN, FMP; $n=125$ ), physician (MD, DO; $n=9$ ), licensed addiction counselor (NAADAC; $\mathrm{n}=71)$, psychologist $(\mathrm{PhD}$, PsyD, EdD; $\mathrm{n}=91$ ), peer provider (CPS, CPRC; $\mathrm{n}=271$ ), no credential $(n=85)$, and other $(n=153)$. There was a higher reporting of a peer provider credential than the number of respondents with experience working as a PSW. We hypothesize that the higher number of peer provider credentials is due to the pressure for clinical supervisors to hold a peer support credential if they are supervising PSWs even if they have never provided direct peer support services themselves.

\section{Instrument}

The 16-item survey included four open-ended questions to assess supervisors' perceptions on: (1) differences supervising PSW as compared to other staff, (2) important qualities 
of PSW supervisors, (3) types of roles when supervising a PSW, and (4) concerns about PSWs in the organization.

\section{Qualitative Data Analysis}

A qualitative content analysis of the responses to the openended items was conducted by three researchers, involving 5 steps: (1) review of the written responses, (2) frequency analysis of terms, (3) pre-coding using NVivo software, (4) coding of individual themes and validation of the coding scheme; and (5) identification of relationships in the data and overarching lessons learned.

Reviews of the responses to the open-ended questions were conducted several times by each of the three researchers, independently, to achieve immersion and obtain a sense of the whole (Tesch, 1990). A conventional content frequency analysis was then performed looking for the most frequently used terms that arose directly from the responses (Hsieh \& Shannon, 2005). Using NVivo, initial pre-codes were developed and entered (Braun \& Clarke, 2012; Morgan, 2018). Coded themes were independently identified, compared, discussed, and verified by two researchers and reviewed by a third researcher to increase the trustworthiness of the analysis and to validate the coding schemes (Patton, 1999). Comparisons were made between what NPS and PS reported and identification of relationships in the data and overarching lessons-learned were recorded. An application to analyze this survey data was submitted to the University Institutional Review Board (IRB) and received exempt status. All survey responses were provided as unidentified data with no link to survey respondents' identifying information.

\section{Findings}

Three major differences in themes emerged from the comparison of responses between NPS and PS; the specific knowledge required of supervisors, understanding of the role of the PSW and supervisors' beliefs about what a PSW needs to do the job. When asked about the differences supervising peer support staff as compared to non-peer staff, $29 \%$ of NPS $(n=157)$ and $42 \%$ of PS $(n=70)$ expressed little or no difference. From both groups, a common emphasis on "treating everyone as an individual" and "maintaining a focus on performance and expectations" emerged. While PS noted there was little difference, their more detailed responses indicated more need for flexibility and a recognition that wellness was relevant to all staff members they supervised, not just PSW. For example, "I see no real differences between other staff. Anyone with a serious illness or disability of any sort requires flexibility, but not lower expectations for performance"; "I do not think it is different, in fact, I think supervisors can learn from the peer support model to discuss the wellness of all coworkers-not just those with a diagnosed mental illness".

\section{Theme 1: Knowledge Required of Supervisors}

Regarding knowledge required of supervisors, 16\% NPS respondents $(n=87)$ indicated a need for supervisors of PSW to have a "working" knowledge of recovery and clarity on how the (non-clinical) PSW role is different from other roles to avoid peer drift. Respondents indicated, "[The supervisor must] be a mentor, teacher, challenger that helps the peer specialist navigate a system that values degrees and licensing."; "Supervisors need to embrace a recovery oriented and strength-based philosophy, have a clinical background (licensed preferred), case manager experience, knowledge/ understanding of the peer role in the recovery process, and give special attention to self-care practices".

Conversely, $21 \%$ of PS $(n=35)$ indicated the importance of their inside knowledge as a peer support provider, leading to a more nuanced understanding of the PSW role by the supervisor. Examples included, "knowing about and also having a lived experience of recovery", "having work experience in the peer support role", and $16 \%$ of PS $(n=27)$ included "having the firsthand understanding of the complexity and emotional burden of the use of lived experience on the job". Generally, PS respondents focused on the importance of the supervisor having lived experience of recovery versus specific knowledge areas to better understand the role of the PSW.

\section{Theme 2: Understanding of the Role of the PSW}

In regard to understanding the PSW role, $20 \%$ of NPS $(n=27)$ indicated a need for greater clarity, particularly on how it is different from other roles. For example, one respondent indicated,

"I think the peer support training should have a manual they need to study, and the supervisor have a copy to guide the peer through it"

Others stated,

"I was asked to supervise our peer supporters with very little guidance about what that meant and what they're expected to do -- which makes it difficult for me to gauge whether or not they're doing what they're expected to do";

"As is often the case, my busy main job duties have made it difficult to research their role in order to feel better equipped for this role - so education on just what peers should be doing/not doing, how their expected boundaries may differ, etc., would be great." 
"I wish, as a supervisor, I was supervised by a trained peer".

$17 \%$ of PS $(n=10)$ expressed greater clarity about the role of the PSW but showed greater concern about a lack of organizational recognition of the complexity and emotional burden of the PSW role than the NPS. Many spoke about co-optation or a drift from the core values that make peer support unique. Responses included, "In my organization the peer role and integrity is very clear. We don't provide clinical services." One respondent stated,

"They [supervisors] need to understand peer support. Too often I have seen clinicians either create role drift for the peer specialist, or (the opposite) they think that the peer specialist has little value. Or, worse yet, they feel that the peer specialist just needs to take their meds and do therapy".

\section{Another respondent indicated,}

"Peer support roles are far more complex than other roles in the $\mathrm{MH}$ field. Being able to supervise peer support workers requires an understanding of the complexity of the role and the ability to help the worker navigate those complexities".

\section{Theme 3: Beliefs About What a PSW Needs to Do the Job}

NPS expressed beliefs that in order to do the job, PSWs require monitoring, self-care, and training on boundaries. When analyzing the beliefs regarding what a supervisor of PSW needs to do the job, $14 \%$ of NPS $(n=75)$ indicated that supervisors felt a responsibility to "monitor the mental health of PSW staff for triggers, signs of decompensation, stress, relapse, and self-care". Several NPS also believed they needed to provide frequent and intensive supportive supervision with a focus on self-care and advocacy when supervising PSW. For example,

"Due to history of illness--requiring them to have a diagnosis and treatment in order to offer them this position, there needs to be understanding of the disorder and it is my opinion that a work WRAP (Wellness Recovery Action Plan) should be in place that can be reviewed in supervision".

Similarly, others said, "Because a Peer staff utilizes their recovery as part of their work duties, then as a supervisor, I feel an obligation to check on how they are managing their wellness"; "They are in a coach role vs treatment role... the boundary for them and all staff can become fuzzy if not outlined and trained ethically so we [ need to] educate PSW staff on professional boundaries"; "Peers need a lot of coaching on issues such as boundaries, confidentiality, conflict of interest, etc.".
$21 \%$ of PS $(n=35)$ focused on the supervisor responsibility for helping PSW as employees to find work/life balance. For example, "Peer staff tend to be very passionate about their work and can have a tendency to overwork themselves which can lead to burnout"; "It is imperative to talk about self-care, burnout, compassion fatigue, and strategies to prevent."; "I also believe that because there is self-disclosure learning how to balance having good boundaries paired with the authenticity that the peer role requires is helpful to talk about".

\section{Discussion}

The themes that emerged from this content analysis add to the understanding of perceptions on supervision from supervisors with experience providing direct peer support services (PS) and supervisors without experience providing direct peer support services (NPS). The responses also support the implementation of a more robust approach to supervision of the peer support workforce. While NPS respondents noted the value of a recovery orientation, PS emphasized the lived experience factor as the primary factor leading to an understanding of recovery. Likewise, PS focused on the importance of lived experience in understanding the role of a PSW, whereas NPS expressed a need for training to understand the role. PS had a strong recognition of the complexities surrounding the peer role and emotional burden related to those complexities and focused on their role of helping the PSW navigate work/life balance. NPS had a more simplified view of the role of the PSW and focused on monitoring of triggers, "decompensation", and relapse. These results point to the natural outcome of PS' lived experience and previous experience working as a PSW influencing how they viewed their role. NPS' clinical expertise appeared to frame how they viewed those they were supervising.

This lean towards a clinical approach by supervisors is born from the 2007 CMS directive that required peer support services to be supervised by a competent mental health practitioner, which in most states is a licensed clinician. To meet this requirement, many organizations billing Medicaid for peer support services defaulted to a model of using NPS to supervise PSW creating a narrative that NPS are the most qualified professionals to supervise PSW (including in situations where Medicaid supervision requirements are not a factor). Given their lack of knowledge and experience with peer support, NPS are in need of training and technical assistance on what peer support is and how to differentiate PSW as colleagues and not patients. Recently, resources have been developed that support both NPS and PS in understanding their role. SAMHSA BRSS TACS created core competencies for PSW in 2016 (SAMSHA, 2016), and the National 
Association of Peer Supporters revised the National Practice Guidelines for Peer Supporters in 2019 to include guidelines on the supervisor's role in guiding PSW to offer services in alignment with their own practice guidelines (N.A.P.S., 2019).

However, additional explicit training that outlines the professional practices and contributions of peers to their nonpeer coworkers is needed to enhance role clarity (Mancini, 2018; Moran et al., 2013). Contrary to casual observation and as reinforced by the PS respondents, peer support is a complex task. Lack of role clarity is not a documented issue in peer support services provided by peer-run organizations where they have been operating under a true apprenticeship model for decades (Gillard, et.al., 2013). The complexities of the peer support role when implemented under clinical direction (in non-peer run behavioral healthcare settings), including lack of clear job descriptions (Cabral, et al., 2014), misunderstanding of the peer support worker's role within existing teams (Cabral et al., 2014; Gillard, et.al., 2013, Kemp, \& Henderson, 2012, Tse, et. al., 2017), and lack of value placed on the contributions of the peer support worker (Asad, \& Chreim, 2016; Gates \& Akabas, 2007; Gates et al., 2010) prevent full integration of PSW in service delivery settings.

As previously noted, a portion (approximately one-third) of respondents expressed that there was very little or no difference between supervising PSW and other staff. However, further examination of the supervisors' perceptions on the specific knowledge required of supervisors, understanding of the role of the PSW and supervisors' beliefs regarding what a PSW needs to do are contrary to this finding. Therefore, the response "no difference" leads to further questions. While PS can offer an apprenticeship level of supervision to PSW staff, how can NPS offer the experiential knowledge required to do the job? One may be able to infer that the response of "no difference" indicates a lack of recognition of the complexity of the PSW role and the need for ongoing mentorship under a skilled and experienced practitioner. Additionally, NPS noted the need for more intensive and frequent supervision of PSW than other staff, while PS demonstrated more willingness to engage in supervision based on mutual learning or a process of co-reflection. Because "no difference" leads to more questions about what is meant without a true understanding of the context of supervision in each respondents' organization or peer support program, this is an area ripe for further study.

Supervision is complex and involves strategies to suit myriad roles and mindsets of manager, coach, mediator, and in some cases, a person who draws on their own lived experience similar to staff they supervise. One way to begin to address the concerns of supervisors, both NPS and PS, is to use a model designed specifically to addresses the unique aspects of supervising the PSW workforce. For example, The Five Critical Functions of Supervision (Edwards, 2018) guides a supervisor to reflect critically on their role and the range and depth of responsibilities. The five functions that comprise the model are: administrative, supportive, educative, advocacy, and evaluative making them both equitable and strengths-based, suggesting that supervision can be employed as compassionate and individualized without being stigmatizing, infantilizing, or punitive. The Five Functions model opens the door to a non-hierarchical evaluation process called co-reflection or co-supervision through which supervisor and supervisee come together as equals to learn with and from each other (Mead, 2014). This model utilizes an accountability tool for supervisors to avoid over-identifying with, or employing punitive measures toward peer support staff, if the supervisor has shared lived experiences with the PSW. The Five Critical Functions of Supervision model recommends opportunities like group supervision, communities of practice, and other collaborative learning circles that allow PSW to learn with and from each other. It also highlights the importance of the supervisor as a collaborator in the process (Table 1).

\section{Limitations}

One limitation of this study is that it was a self-report by supervisors about their own supervisory role. Self-report tends to have a halo effect, under which respondents have the desire to "look good." Responses do not include comparative or contrasting perspectives of the PSWs receiving supervision. Another limitation of this study is the lack of distinction between responses from supervisors practicing in substance use recovery treatment settings versus those is mental health provider agencies. In some states, mental health and substance use treatment are integrated in a single setting. However there remain fundamental differences in

Table 1 Comparisons of NPS and PS supervisor responses

\begin{tabular}{llc}
\hline Question & NPS Responses & PS Responses \\
\hline In what ways, if any, are supervising peer support workers different from other staff? & $N=524$ & $N=163$ \\
What qualities are important for a supervisor of peer support workers to have? & $N=531 \quad N=533 \quad N=167$ \\
What are the different roles you play when supervising a peer support worker? & $N=130 \quad N=59$ \\
Write any questions or concerns you have about the peer support workers in your organizations & $N=167$ \\
\hline
\end{tabular}


approach to both treatment types and the resulting peer support which could impact supervision and perceptions of the role of the supervisor. Finally, an inability to ask respondents to clarify answers such as, "there is little or no difference between supervising PSW and other staff" can be seen as a limitation.

\section{Conclusion}

Many organizations indicate a lack of adequate supervision as a barrier to offering peer support services (Kemp, \& Henderson, 2012; Vandewalle, et al., 2016) and the impacts of this can be cooptation of the peer role, PSW treated as second-class professionals, employee dissatisfaction, dissatisfaction by those receiving services, high turnover, disruption in service, and the potential for discontinuation of peer support services. The increased use of NPS has given rise to a common theme in the literature that there is confusion and a lack of role clarity for the PSW position; a theme that does not appear in the literature specific to peer-run organizations and peer support communities, which operate under an authentic apprenticeship model. This role confusion is not surprising given that NPS lack the experience that PSW are expected and required to have. As the peer support workforce grows, the challenge of who and how to supervise PSW will also proliferate.

Organizations best suited to maintain peer support programs that avoid role confusion and provide professional development to PSW will be those that adopt PS as the model for supervision, who utilize a co-model of both PS and NPS when a licensed supervisor is required, and/or who engage in peer-led, collective co-reflection through group supervision or in communities of practice (Cronise, 2016). While these options most align with the apprenticeship model, there will be organizations with limited financial or human resources further necessitating the adoption of universal competencies and guidelines relevant to both NPS and PS such as the N.A.P.S. National Practice Guidelines for Supervisors. The knowledge and understanding a supervisor holds in relation to their role and the role of the PSW will be critical in developing appropriate training and resources for both NPS and PS. Undoubtedly, supervisors' beliefs about peer support and their practices will play a defining role in the future direction of the peer support workforce.

Acknowledgements The authors thank Cheryl Gagne, of BRSS TACS [7] for her support in developing and reviewing the survey language prior to dissemination, and gratefully acknowledge the sponsorship of the State of Florida, Agency for Health Care Administration, which gave permission to Magellan to share the results of these analyses. The findings and conclusions described herein are those of the authors and do not necessarily represent the positions of Magellan Complete Care,
Inc., the State of Florida, Agency for Health Care Administration, or other institutions with which the authors are affiliated.

Funding The authors have no other financial disclosures to report.

\section{References}

Asad, S., \& Chreim, S. (2016). Peer support providers' role experiences on interprofessional mental health care teams: A qualitative study. Community Mental Health Journal, 52, 767-774.

Braun, V., \& Clarke, V. (2012). Thematic analysis. Research designs: Quantitative, qualitative, neuropsychological, and biologicalln $\mathrm{H}$. Cooper, P. M. Camic, D. L. Long, A. T. Panter, D. Rindskopf, \& K. J. Sher (Eds.), APA handbook of research methods in psychology (Vol. 2, pp. 57-71). American Psychological Association.

Cabral, L., Strother, H., Muhr, K., Sefton, L., \& Savageau, J. (2014). Clarifying the role of the mental health peer specialist in Massachusetts, USA: Insights from peer specialists, supervisors and clients. Health and Social Care in the Community, 22(1), 104-112.

Collins, A. (2006). Cognitive apprenticeship. In R. K. Sawyer (Ed.), The Cambridge handbook of the learning sciences (pp. 47-60). Cambridge University Press.

Cronise, R. (2016). Collaborative learning: A next step in the training of peer support providers. Psychiatric Rehabilitation Journal, 39(3), 292-294.

Daniels, A. S., Tunner, T. P., Powell, I., Fricks, L., Ashenden, P. (2015) Pillars of peer support-VI: peer specialist supervision. Retrieved from: www.pillarsofpeersupport.org. Accessed December 2020.

DBHIDS, (2017). Peer support toolkit. https://dbhids.org/peer-suppo rt-toolkit/. Accessed December 2020.

Delman, J., \& Klodnick, V. (2016). Factors supporting the employment of young adult peer providers: Perspectives of peers and supervisors. Community Mental Health Journal, 53(7), 1-11.

Donaldson, A. L. (2015). Pre-professional training for serving children with ASD: An apprenticeship model of supervision. Teacher Education and Special Education., 38(1), 58-70. https://doi.org/10. $1177 / 0888406414566995$

Edwards, J. (2018). Five critical functions of supervising peer supporters, supervision track: An introduction to supervising peer specialists, key concepts. Academy of Peer Services.

Feinstein, R., Huhn, R., \& Yager, J. (2015). Apprenticeship model of psychotherapy training and supervision: Utilizing six tools of experiential learning. Academic Psychiatry, 39(5), 585-589. https://doi.org/10.1007/s40596-015-0280-6

Gates, L., \& Akabas, S. (2007). Developing strategies to integrate peer providers into the staff of mental health agencies. Administrative Policy Mental Health and Mental Health Services Research, 34, 293-306.

Gates, L., Mandiberg, J., \& Akabas, S. (2010). Building capacity in social service agencies to employ peer providers. Psychiatric Rehabilitation Journal, 2, 145-152.

Gillard, S. G., Edwards, C., Gibson, S. L., Owen, K., \& Wright, C. (2013). Introducing peer worker roles into UK mental health service teams: A qualitative analysis of the organisational benefits and challenges. BMC Health Services Research, 13(1), 1-13.

Hartley, B., Courtney, W., Rosswum, M., \& LaMarca, V. (2016). The apprentice: An innovative approach to meet the behavior analysis certification board's supervision standards. Behavioral Analysis Practice, 9(4), 329-338.

Hendry, P., Hill, T., \& Rosenthal, H. (2014). Peer services toolkit: A guide to advancing and implementing peer-run behavioral health services. ACMHA: The College for Behavioral Health Leadership and Optum. 
Hsieh, H., \& Shannon, S. (2005). Three approaches to qualitative content analysis. Qualitative Health Research, 15(9), 1277-1288.

Jenkins, S., Chenneville, T., \& Salnaitis, C. (2017). Are peer specialists happy on the job? Brief Report Psychiatric Rehabilitation Journal, 41(1), 72-75.

Jorgenson, J., \& Schmook, A. (2014). Enhancing the peer provider workforce: Recruitment, supervision, and retention. National Association of State Mental Health Program Directors.

Kemp, V., \& Henderson, A. R. (2012). Challenges faced by mental health peer support workers: Peer support from the peer support's point of view. Psychiatric Rehabilitation Journal, 30(4), 337-340.

Kuhn, W., Bellinger, J., Stevens-Manser, S., \& Kaufman, L. (2015). Integration of peer specialists working in mental health service settings. Community Mental Health Journal, 51, 453-458.

Mancini, M. (2018). An exploration of factors that effect the implementation of peer support services in community mental health settings. Community Mental Health Journal, 54, 127-137.

Martin, E., Jordan, A., Razavi, M., Van Burnham, I.V. (2017). Systematic review of the literature: Identifying top 25 core competencies of SUD peer supervisors. Retrieved from: https://www.oregon. gov -> 6-23-2017-PDS-Supervisor-Core-Comps-SupervisionSystematic\%20Review-Summary-12-2016.pdf. Accessed December 2020

Mead, S. (2014). Intentional peer support co-reflection guide. Intentional peer support: An alternative approach.Intentional peer support.

Morgan, D. (2018). Themes, theories, and models. Qualitative Health Research, 28(3), 339-345.

Moran, G. S., Russinova, Z., Vasudha, G., \& Gagne, C. (2013). Challenges experienced by paid peer providers in mental health recovery: A qualitative study. Community Mental Health Journal, 49, 281-291. https://doi.org/10.1007/s10597-012-9541-y

National Association of Peer Supporters (2019). National Practice Guidelines for Peer Specialists and Supervisors. (Formerly known as the International Association of Peer Supporters, N.A.P.S.). Retrieved from: https://www.peersupportworks.org/wp-conte nt/uploads/2021/02/nationalguidelines_updated.pdf. Accessed December 2020.

Patton, M. (1999). Enhancing the quality and credibility of qualitative analysis. HSR: Health Science Research, 34(5), 1189.

SAMHSA. (2016). Core Competencies for Peer Support Workers. Substance Abuse and Mental Health Services Administration Bringing Recovery Supports to Scale, Technical Assistance Center Strategy (BRSS TACS). Retrieved from https://www.samhsa.gov/ brss-tacs/recovery-support-tools/peers/core-competencies-peerworkers. Accessed December 2020.
Smith, D. (2007). Letter from CMS Director Dennis Smith to the State Medicaid Directors with Guidance on the Development of Peer Support Services Under Medicaid. August 15, 2007. Dept. of Health and Human Services, SMDL-\#07-11. Retrieved from https://downloads.cms.gov/cmsgov/archived-downloads/SMDL/ downloads/SMD081507A.pdf

Stalmeijer, R., Dolmans, D., Snellen-Balendong, H., Santen-Hoeufft, H., Wolfhagen, I., \& Scherpbier, A. (2013). Clinical teaching based on principles of cognitive apprenticeship: Views of experienced clinical teachers. Academic Medicine: Journal of the Association of American Medical Colleges. https://doi.org/10.1097/ ACM.0b013e31828fff12

Stephan, A., \& Cheung, G. (2017). Clinical teaching and supervision in postgraduate psychiatry training: The trainee perspective. Australia Psychiatry, 25(2), 191-197.

Stoltenberg, C. (2005). Enhancing professional competence through developmental approaches to supervision. American Psychologist, 60(8), 857-864.

Swarbrick, M. (2010). Peer wellness coaching supervisor manual. Institute for Wellness and Recovery Initiatives.

Tesch, R. (1990). Qualitative research: Analysis types and software tools. Falmer Press.

Tse, S., Mak, W. W. S., Lo, I. W. K., Liu, L. L., Yuen, W. W. Y., Yau, S., Ho, K., Chan, S. K., \& Wong, S. (2017). A one-year longitudinal qualitative study of peer support services in a non-Western context: The perspectives of peer support workers, service users, and co-workers. Psychiatry Research, 255, 27-35.

Tucker, S. J., Tiegreen, W., Toole, J., Banathy, J., Mulloy, D., \& Swarbrick, M. (2013). Supervisor guide: Peer support whole health and wellness. Decatur, GA: Georgia Mental Health Consumer Network.

Vandewalle, J., Debyser, B., Beeckman, D., Vandecasteele, T., Van Hecke, A., \& Verhaeghe, S. (2016). Peer workers' perceptions and experiences of barriers to implementation of peer worker roles in mental health services: A literature review. International Journal of Nursing Studies, 60, 234-250.

Wolf, J., Lawrence, L., Ryan, P., \& Hoge, M. (2010). Emerging practices in employment of persons in recovery in the mental health workforce. American Journal of Psychiatric Rehabilitation, 13, 189-207.

Publisher's Note Springer Nature remains neutral with regard to jurisdictional claims in published maps and institutional affiliations. 